\title{
DESARROLLO A DISTANCIA DE LA MAESTRÍA EN DOCENCIA \\ PARA LA EDUCACIÓN MEDIA SUPERIOR (MADEMS) DE LA UNIVESIDAD NACIONAL AUTÓNOMA DE MÉXICO (UNAM)
}

\author{
(DISTANCE EDUCATION PROGRAM FOR A MASTER'S DEGREE ON TEACHERS EDUCATION AT \\ THE HIGH SCHOOL LEVEL ORGANIZED BY UNAM (NATIONAL UNIVERSITY OF MEXICO)
}

\author{
Francisco Javier Sierra Vázquez \\ Jorge Barojas Weber \\ Ofelia Contreras Gutiérrez \\ Rina María Martínez Romero \\ Universidad Nacional Autónoma de México, UNAM (México)
}

\section{RESUMEN}

Describimos las características generales de la MADEMS y las acciones estratégicas necesarias para impartir la modalidad a distancia. También comentamos sus posibilidades de generalización y colaboración con centros de educación media y superior en México y en otros países, así como las implicaciones que tiene este posgrado en el marco de los espacios educativos en los cuales participa la institución, en tanto modelo de programa multidisciplinario, experiencia de efecto multiplicador y proyecto donde convergen aportaciones a la educación, la capacitación, la innovación, la cooperación y el desarrollo.

\begin{abstract}
The general characteristics of the Master's degree program MADEMS and the strategic actions required to organize the distance education program are described. The possibilities for generalization and collaboration with high schools and colleges in Mexico and in other countries are examined. Furthermore, the implications of this program in the context of educational spaces in which our institution is involved are also considered. This is done by taking into account that this program provides a multidisciplinary model with a multiplicative effect and that it is also a project which contributes to education, training, innovation, cooperation and development.
\end{abstract}


Francisco Sierra, Jorge Barojas, Ofelia Contreras y Rina MartíneZ

DESARROLLO A DISTANCIA DE LA MAESTRÍA EN DOCENCIA PARA LA EDUCACIÓN MEDIA SUPERIOR (MADEMS) DE LA UnivERSidAd NACIONAL AUTÓNOMA DE MÉXico (UNAM)

\section{INTRODUCCIÓN}

Una componente fundamental de la problemática del bachillerato de la UNAM, en sus dos subsistemas, el Colegio de Ciencias y Humanidades y la Escuela Nacional Preparatoria, es la falta de preparación formal de sus profesores para la docencia, tanto en el área de las ciencias de la educación, como en el área en que se ejerce la docencia. Esta situación es extensiva a la mayoría de las instituciones educativas del país, en lo que respecta al nivel de la Educación Media Superior (EMS). Sin embargo, pese a sus logros y avances, la EMS se caracteriza por los bajos porcentajes de aprobación y eficiencia terminal de alumnos que cuando egresan, manifiestan deficiencias en sus capacidades para aprender y actualizarse por su cuenta. Además, en este nivel educativo existen grandes heterogeneidades y dispersiones curriculares, a todo lo cual se agrega un crecimiento importante en la demanda educativa para las siguientes décadas, complicada por el hecho de que está por jubilarse un número significativo de profesores actualmente en ejercicio.

La Maestría en Docencia para la Educación Media Superior (MADEMS) se inició en la modalidad escolarizada el 9 de febrero de 2004 en ocho campos de conocimiento: Biología, Ciencias Sociales, español, Filosofía, Física, Historia, Matemáticas y Química. En este posgrado colaboran nueve entidades académicas participantes: las Facultades de Ciencias, Ciencias Políticas y Sociales, Estudios Superiores Acatlán, Estudios Superiores Iztacala, Filosofía y Letras, Psicología y Química, y los Institutos de Investigaciones Filológicas y Matemáticas; así como dos entidades de apoyo: la Escuela Nacional "Colegio de Ciencias y Humanidades" y la "Escuela Nacional Preparatoria".

La MADEMS es un programa de programas, profesionalizante, orientado a la formación de profesores del nivel de EMS y referido a múltiples campos de conocimiento. Para dar comienzo a la Maestría se han seleccionado campos que corresponden a disciplinas básicas y obligatorias comprendidas en los planes de estudio de la EMS y que presentan altos índices de reprobación (las ocho indicadas con anterioridad), con la perspectiva de ampliarla a todas las asignaturas que se ofrecen en la EMS. Dada la urgencia de atender este proceso de capacitación a nivel nacional, el desarrollo a distancia de este posgrado es una alta prioridad, que empezará a ser atendida próximamente a nivel piloto, en el campo de conocimiento de la Biología, en la Facultad de Estudios Superiores Iztacala. 
Francisco Sierra, Jorge Barojas, Ofelia Contreras y Rina Martínez

DESARROLLO A DISTANCIA DE LA MAESTRÍA EN DOCENCIA PARA LA EDUCACIÓN MEDIA SUPERIOR (MADEMS) DE LA UnivERSIDAD NACIONAL AUTÓNOMA DE MÉXICO (UNAM)

\section{DESCRIPCIÓN DE LA MADEMS}

Esta sección contiene información tomada del documento de presentación de la MADEMS; para mayores detalles, consultar el sitio: http://www.posgrado.unam. $\mathrm{mx} /$ madems/index.html .

La MADEMS ha sido diseñada con cuatro características básicas:

1. Participación multidisciplinaria e institucional. Un rasgo singular de este posgrado ha sido la participación de las once entidades académicas ya mencionadas. Además, en su diseño y organización participó en forma primordial la Dirección General de Estudios de Posgrado.

2. Docencia como profesión. Esta concepción del quehacer docente obliga a que la Maestría ponga énfasis en la formación práctica del alumno en condiciones de trabajo en aula, en relación con las tareas que el futuro docente deberá tener para el desarrollo de su actividad y el tiempo, los recursos y la infraestructura para llevar a cabo su quehacer frente a grupo.

3. Concepción integral de un currículo flexible. La Maestría ofrece una preparación sustentada en tres líneas de formación (la socio-ético-educativa, la psicopedagógico-didáctica y la disciplinaria), con el fin de habilitar a los alumnos para una enseñanza efectiva sobre un campo específico de conocimientos de alguna de las materias de la EMS.

4. Programa de posgrado de calidad. La calidad del programa se garantiza aplicando mecanismos para el seguimiento y evaluación de: (a) la funcionalidad eficiente y eficaz de la organización académico administrativa de la Maestría a través de la operación de su Comité Académico; (b) el funcionamiento del sistema tutoral; (c) la obtención del grado bajo la figura de tesis que deberá corresponder a un proyecto de aplicación docente o de interés profesional, de acuerdo con los objetivos del Programa; (d) la participación colegiada en la conformación de campos de conocimiento y su continua expansión; (e) los procedimientos de selección de los alumnos; y (f) la evaluación bianual del funcionamiento de toda la Maestría.

El plan de estudios de esta Maestría es mixto ya que está conformado por tres ámbitos docentes:

1. El ámbito de la Docencia General referida al tronco común que cursan todos los estudiantes y que comprende cinco actividades académicas correspondientes a dos líneas de formación, la socio-ético-educativa y la psicopedagógico-didáctica; 
Francisco Sierra, Jorge Barojas, Ofelia Contreras y Rina MartíneZ

DESARROLLO A DISTANCIA DE LA MAESTRÍA EN DOCENCIA PARA LA EDUCACIÓN MEDIA SUPERIOR (MADEMS) DE LA

UNIVERSIDAD NACIONAL AUTÓNOMA DE MÉXICO (UNAM)

2. El ámbito de Docencia Disciplinaria, comprende también cinco actividades académicas y se refiere a la profundización y dominio tanto del campo de conocimiento respectivo como de su didáctica, según sea el origen de las licenciaturas de las cuales provengan los estudiantes y la disciplina a la cual estará asociada su práctica profesional como docentes de la EMS, y

3. El ámbito de la Integración de la Docencia en donde se conjugan los aprendizajes de las docencias antes mencionadas, la General y la Disciplinaria, y se hace énfasis en el trabajo de elaboración de la tesis a través de cinco actividades académicas: un seminario, un taller y tres prácticas docentes supervisadas.

Conviene observar que la complejidad operativa de la MADEMS se debe a los siguientes diez factores:

(1) las entidades académicas del programa (once en el momento inicial);

(2) los campos de conocimiento propios de la Maestría (los ocho antes indicados);

(3) los doce Programas de Posgrado conectados con los campos de conocimiento en los cuales se ofrece la Maestría, involucrados en la revisión y evaluación continua de los planes de estudios y en la impartición de algunas actividades académicas de los paquetes de optativas disciplinarias correspondientes a avances y desarrollos del campo de conocimiento respectivo(Ciencias Biológicas, Ciencias Físicas, Ciencias Matemáticas, Ciencias Políticas y Sociales, Ciencias Químicas, Filosofía, Filosofía de la Ciencia, Historia, Letras, Lingüística, Pedagogía y Psicología).

(4) las diversas actividades académicas del plan de estudios que corresponden a un total de 15 actividades a lo largo de cuatro semestres, 6 de las cuales forman parte de paquetes de optativas, sumando un total de 85 actividades académicas diferentes para los ocho campos de conocimiento iniciales;

(5) los campi de la UNAM en donde se organizarán dichas actividades (empezamos en Acatlán, Ciudad Universitaria e Iztacala);

(6) la diversidad en cuanto a formación inicial y experiencia docente de los alumnos aceptados luego de haber aprobado los exámenes de admisión y pasar satisfactoriamente la entrevista con los correspondientes comités de admisión en cada campo de conocimiento (actualmente se encuentran inscritos 400 alumnos que corresponden a cinco generaciones);

(7) las posibles modalidades de impartición de la MADEMS: la presencial con la cual empezamos y a distancia, con la cual esperamos operar en agosto del 2005; 
Francisco Sierra, Jorge Barojas, Ofelia Contreras y Rina MartíneZ

DESARROLLO A DISTANCIA DE LA MAESTRÍA EN DOCENCIA PARA LA EDUCACIÓN MEDIA SUPERIOR (MADEMS) DE LA UnivERSidAd NACIONAL AuTÓNOMA DE MÉXICO (UNAM)

(8) el alcance del programa, inicialmente restringido a la zona metropolitana, pero con posibilidades de alcanzar una cobertura que podrá ser de nivel nacional e inclusive regional;

(9) la periodicidad con la cual se publique la convocatoria para inscripciones, sea semestral o anual, y

(10) el hecho de que la MADEMS sirve de enlace entre los tres ciclos de formación universitaria: el profesional (nivel de ingreso de los mismos estudiantes), el posgrado (nivel de egreso de los estudiantes de la MADEMS) y el bachillerato (nivel de la práctica docente de los egresados de la Maestría).

\section{LA MADEMS A DISTANCIA}

La sociedad del conocimiento múltiple y del aprendizaje continuo reclama que la formación de profesionales sea de alta calidad educativa y que integre de forma adecuada nuevos métodos y modalidades didácticas. Ejemplo de ello es el destacado desarrollo mundial que se vive actualmente de la Educación Universitaria a Distancia. Para que esta modalidad educativa promueva el aprendizaje significativo de los alumnos es indispensable identificar dos ámbitos claramente diferenciados: las actividades de aprendizaje que se realizarán a distancia y las que se requieren semi-presenciales o presenciales.

El concepto básico de la educación a distancia implica que maestros y alumnos están separados por una distancia y en ocasiones por el tiempo; por lo que es necesario introducir un sistema de comunicación que permita el intercambio de información y proporcione un canal de interacción. Sin embargo, de acuerdo con Moore y Kearsley (1996), la distancia no solo se refiere a la separación física entre alumnos y maestros, sino a la distancia en la comprensión y percepción. Y además, esta distancia también implica una independencia potencial en los estudiantes para dirigir y controlar su aprendizaje. Como resultado de esta característica, se necesitan técnicas especiales de diseño instruccional, una plataforma tecnológica que le dé soporte a la comunicación y un sistema de administración y organización propio de esta modalidad.

De aquí se desprende que a la complejidad intrínseca de la MADEMS como programa multidisciplinario de posgrado, habría que sumar las características: las dificultades derivadas de que los alumnos no están familiarizados con esta modalidad educativa; la población potencial de alumnos es grande y diversa, y necesita un número suficiente de tutores; el diseño instruccional debe tener una estructura que facilite a los alumnos tomar decisiones acerca de qué, cuándo, cómo, dónde y cuánto 
Francisco Sierra, Jorge Barojas, Ofelia Contreras y Rina MartíneZ

DESARROLLO A DISTANCIA DE LA MAESTRÍA EN DOCENCIA PARA LA EDUCACIÓN MEDIA SUPERIOR (MADEMS) DE LA UnivERSidAd NACIONAL AUTÓNOMA DE MÉXico (UNAM)

estudiar; los autores de los cursos y expertos en los contenidos deben tomar en cuenta ambientes de aprendizaje que no son iguales a los que se tienen en la modalidad presencial; los autores y los tutores necesitan canales adicionales de comunicación que propicien la coherencia de los cursos que se impartan; y un largo y cambiante etcétera propio de la novedad de esta modalidad.

El cumplimiento de los objetivos de aprendizaje en este modelo "mixto" de educación a distancia requiere valorar apropiadamente el hecho de que las actuaciones de los alumnos y de los docentes-tutores no deben limitarse a un solo ámbito educativo. De esta forma y partiendo de esta premisa básica, podemos destacar la importancia de llevar a cabo las mejores prácticas educativas que en gran medida aseguren que los alumnos aprendan, es decir a través de diversos ambientes o entornos para el aprendizaje: virtuales y/o presenciales (Barberá, 2000; Pozo y Monereo, 1999;)

Desde la perspectiva de la estrategia para alcanzar esta meta, hemos tomado en cuenta las siete etapas que proponen Bates y Poole (2003):

1. Definir los objetivos de aprendizaje. En nuestro caso son los mismos objetivos de la modalidad presencial de la MADEMS.

2. Seleccionar la tecnología adecuada. Hemos optado por el desarrollo de una plataforma propia para Internet.

3. Diseñar los cursos. El diseño instruccional considera las necesidades de los alumnos y los tutores para el trabajo en Internet.

4. Desarrollar los cursos. El punto de partida ha sido el material que se tiene para la modalidad presencial y se está adaptando para su uso en la plataforma tecnológica.

5. Impartir los cursos. Iniciamos esta etapa en febrero de 2006 en el área de Biología.

6. Evaluar los cursos.

7. Revisar los cursos.

En nuestro caso las etapas dosytres son interdependientes y se están desarrollando simultáneamente. Y debido a la amplia gama de instrumentos de comunicación que se pueden ofrecer a través de la Internet, hemos escogido esta tecnología para desarrollar la MADEMS a distancia.

Uno de los elementos más críticos para definir la plataforma tecnológica ha sido la elección de la forma de abordar los procesos de enseñanza-aprendizaje. De 
Francisco Sierra, Jorge Barojas, Ofelia Contreras y Rina Martínez

DESARROLLO A DISTANCIA DE LA MAESTRÍA EN DOCENCIA PARA LA EDUCACIÓN MEDIA SUPERIOR (MADEMS) DE LA UnivERSIDAD NACIONAL AuTÓNOMA DE MÉXico (UNAM)

acuerdo con la metodología convencional, autores como Harasim, Hiltz, Teles y Turoff (1995), proponen una serie de enfoques, estructuras y recursos tecnológicos para ambientes virtuales de aprendizaje en el sentido en el que Lévy (1995) define la virtualidad; es decir, no como una manera de ser opuesta a lo real, no como lo opuesto del aprendizaje en el aula, sino como una mutación del aprendizaje que lo redefine y lo actualiza.

En toda modalidad educativa resulta prioritario distinguir tanto los agentes que participan en el complejo proceso de enseñanza-aprendizaje, como los medios y recursos que utilizan (García Aretio, 1999; Bautista Liébana, Martínez Romero y Sáinz Ibáñez, 2001; González Boticario, Gaudioso Vázquez, Hernández del Olmo, 2000; Bartolomé, 1999; Aguirre Ocaña, 1996).

Dada la diversidad de agentes que componen el modelo de educación a distancia de la MADEMS y de los factores que caracterizan su complejidad operativa, tales como los señalados al final de la sección II, para que las interacciones entre tales agentes sean exitosas deben coordinarse y llevarse un seguimiento de forma permanente que permita precisar en qué medida se cumplen los objetivos de aprendizaje de la maestría y es efectiva la participación activa de todos sus agentes, en particular de los alumnos (Barberá, Badia y Mominó, 2001; Feldman, McElroy y LaCour, 2000).

Hemos definido una estructura para el Campus Virtual de la MADEMS que toma en cuenta dos hechos de nuestra realidad presente: no hay un número suficiente de expertos para cubrir las necesidades tutorales y la modalidad a distancia permite atender a una gran población de alumnos más allá de la zona metropolitana de la Ciudad de México. Esta situación nos lleva a iniciar la operación de la MADEMS a distancia con tutores que son alumnos avanzados de la Maestría y tienen la capacidad para orientar a los alumnos en su aprendizaje a distancia. Para ello se han definido diferentes agentes y funciones que permiten organizar la actividad académica desde el diseño de los cursos hasta su impartición y evaluación. A continuación se muestra una tabla con la descripción de estas funciones.

\begin{tabular}{|l|l|}
\hline \multicolumn{1}{|c|}{ AGENTE } & \multicolumn{1}{|c|}{ FUNCIÓN } \\
\hline Administrador & $\begin{array}{l}\text { Establecer y configurar los recursos tecnológicos y } \\
\text { privilegios de los usuarios de la plataforma. }\end{array}$ \\
\hline Coordinador & $\begin{array}{l}\text { Organizar el acceso de los usuarios y la información que es } \\
\text { común para todos los cursos. }\end{array}$ \\
\hline
\end{tabular}


Francisco Sierra, Jorge Barojas, OfElia Contreras y Rina Martínez

DESARROLLO A DISTANCIA DE LA MAESTRÍA EN DOCENCIA PARA LA EDUCACIÓN MEDIA SUPERIOR (MADEMS) DE LA UnivERSidAd NACIONAL AUTÓNOMA DE MÉXico (UNAM)

\begin{tabular}{|l|l|}
\hline Autor & $\begin{array}{l}\text { Realizar el diseño instruccional, aportar los materiales } \\
\text { didácticos y organizar las actividades de aprendizaje y los } \\
\text { recursos del curso. }\end{array}$ \\
\hline Monitor & $\begin{array}{l}\text { Supervisar la operación de la plataforma desde las } \\
\text { perspectivas tecnológica y educativa. }\end{array}$ \\
\hline Tutor(es) & Orientar y evaluar el aprendizaje de los alumnos. \\
\hline Alumnos & Realizar las actividades de aprendizaje y evaluación. \\
\hline
\end{tabular}

Desde el punto de vista de los materiales que utilizan los agentes de la MADEMS a distancia, los tres materiales básicos que conformarán cada asignatura son las Guías didácticas, las Unidades de aprendizaje y la Guía del instructor. Esto se aplica a cada uno de los diferentes módulos que integran la maestría, tal como se muestra en la Tabla anexa:

\begin{tabular}{|l|l|}
\hline \multicolumn{1}{|c|}{ MÓDULO } & \multicolumn{1}{c|}{ CONTENIDOS } \\
\hline 1. Socio-ético & $\begin{array}{l}\text { Historia, Sociedad y Educación } \\
\text { Optativa socioeducativa: Sistemas de Educación Me- } \\
\text { dia Superior } \\
\text { Optativo ético-educativa: Ética de la práctica profe- } \\
\text { sional }\end{array}$ \\
\hline 2. Psicopedagógico & $\begin{array}{l}\text { Desarrollo del Adolescente } \\
\text { Psicopedagogía de la Enseñanza y el Aprendizaje } \\
\text { Optativa de apoyo al desarrollo de tesis: tecnología } \\
\text { educativa }\end{array}$ \\
\hline 3. Didáctico & $\begin{array}{l}\text { Optativa disciplinaria I: Fundamentos Teórico-met- } \\
\text { odológicos } \\
\text { Didáctica de la Disciplina I } \\
\text { Didáctica de la Disciplina II }\end{array}$ \\
\hline 4. Disciplinario & $\begin{array}{l}\text { Optativa disciplinaria II: Avances y desarrollos de la } \\
\text { Disciplina I } \\
\text { Optativa disciplinaria III: Avances y desarrollos de la } \\
\text { Disciplina II }\end{array}$ \\
\hline 5. Integración práctica & $\begin{array}{l}\text { Práctica Docente I } \\
\text { Práctica Docente II } \\
\text { Práctica Docente III } \\
\text { Seminario de integración para el trabajo de grado }\end{array}$ \\
\hline
\end{tabular}


Francisco Sierra, Jorge Barojas, Ofelia Contreras y Rina MartíneZ

DESARROLLO A DISTANCIA DE LA MAESTRÍA EN DOCENCIA PARA LA EDUCACIÓN MEDIA SUPERIOR (MADEMS) DE LA UnivERSIDAD NACIONAL AUTÓNOMA DE MÉXICO (UNAM)

Asimismo, cabe señalar que los materiales básicos, estarán ampliamente complementados por diversos recursos y medios, tales como: materiales impresos, materiales multimedia en discos compactos y en Internet, portafolios electrónicos y participación en grupos y foros virtuales (Uden, 2003; García Aretio, 1998; Bautista Liébana, Martínez Romero y Sáinz Ibáñez, 2001; Spector, 2001).

En esencia, la plataforma que estamos desarrollando para la MADEMS a distancia es del tipo LCMS (Learning Content Management System) y está constituida por tres subsistemas: La Sección Escolar, las Aulas Virtuales y la Agenda.



Fig. 1 Los tres subsistemas de la MADEMS virtual

La Sección Escolar permite asignar las claves de acceso y contraseñas de seguridad a los usuarios del sistema, así como establecer los grupos para cada asignatura. Dentro de este subsistema también se tienen los servicios de información para los alumnos que se muestran en la fig. 2:

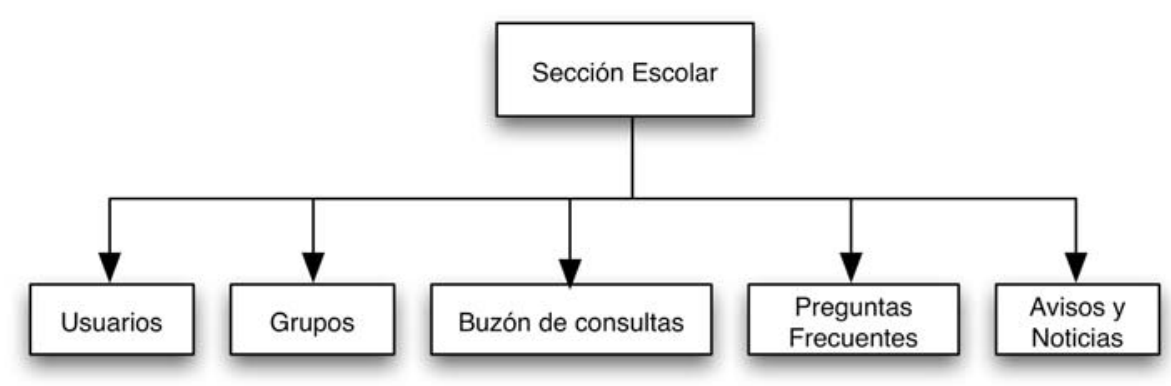

Fig. 2 La Sección Escolar 
Francisco Sierra, Jorge Barojas, OfElia Contreras y Rina MarTíneZ

DESARROLLO A DISTANCIA DE LA MAESTRÍA EN DOCENCIA PARA LA EDUCACIÓN MEDIA SUPERIOR (MADEMS) DE LA UNIVERSIDAD NACIONAL AUTÓNOMA DE MÉXICO (UNAM)

Para cada grupo de alumnos de cada asignatura se tiene un aula virtual, cuya estructura se muestra en la fig. 3 .

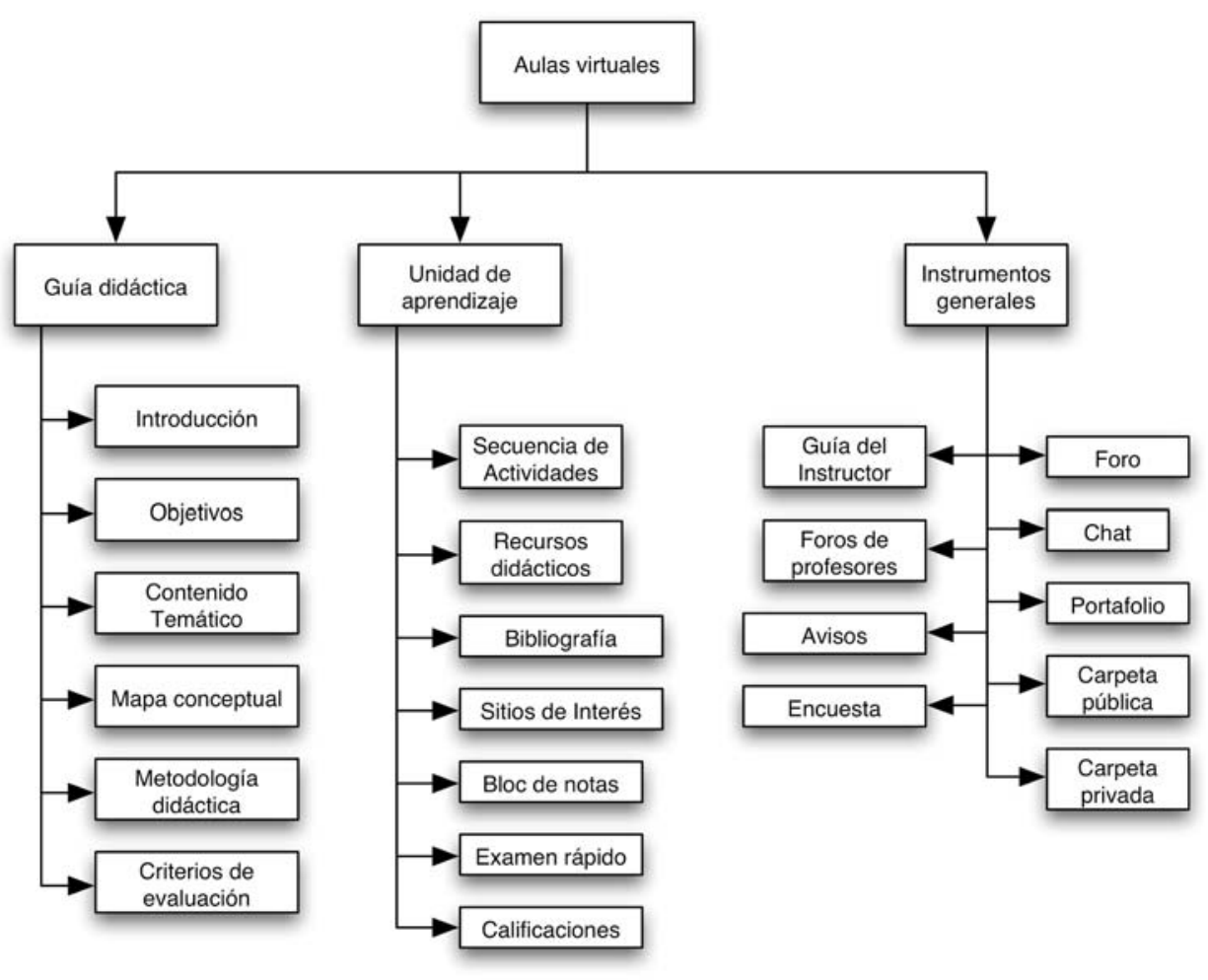

Fig. 3 Estructura de las aulas virtuales

La Guía didáctica ofrece la información necesaria y suficiente para que los alumnos tomen las decisiones pertinentes a su aprendizaje. Cada Unidad de Aprendizaje comprende los materiales de estudio y las actividades que los alumnos deben realizar, tanto de forma individual como en colaboración en grupos pequeños. Además, se ofrece: una bibliografía complementaria, ligas a sitios de interés, instrumentos de autoevaluación y un sistema de control de calificaciones parciales. Cada aula virtual tiene acceso a una guía para el instructor con los antecedentes del curso y recomendaciones sobre metodología didáctica, actividades de aprendizaje, actividades de evaluación, materiales de aprendizaje, trabajos y proyecto final. También contiene los instrumentos de comunicación como foros y chat para el 
Francisco Sierra, Jorge Barojas, Ofelia Contreras y Rina Martínez

DESARROLLO A DISTANCIA DE LA MAESTRÍA EN DOCENCIA PARA LA EDUCACIÓN MEDIA SUPERIOR (MADEMS) DE LA UnivERSIDAD NACIONAL AUTÓNOMA DE MÉXICO (UNAM)

trabajo en grupos y la comunicación entre tutores; así como una sección de avisos, un sistema de carpetas para guardar documentos, un portafolio para que cada alumno entregue los documentos que hacen evidente su aprendizaje y un instrumento para encuestas rápidas que le facilitan al tutor tomar decisiones.

Finalmente, se cuenta con una agenda que permite programar los cuatro tipos de eventos indicados en la fig. 4.

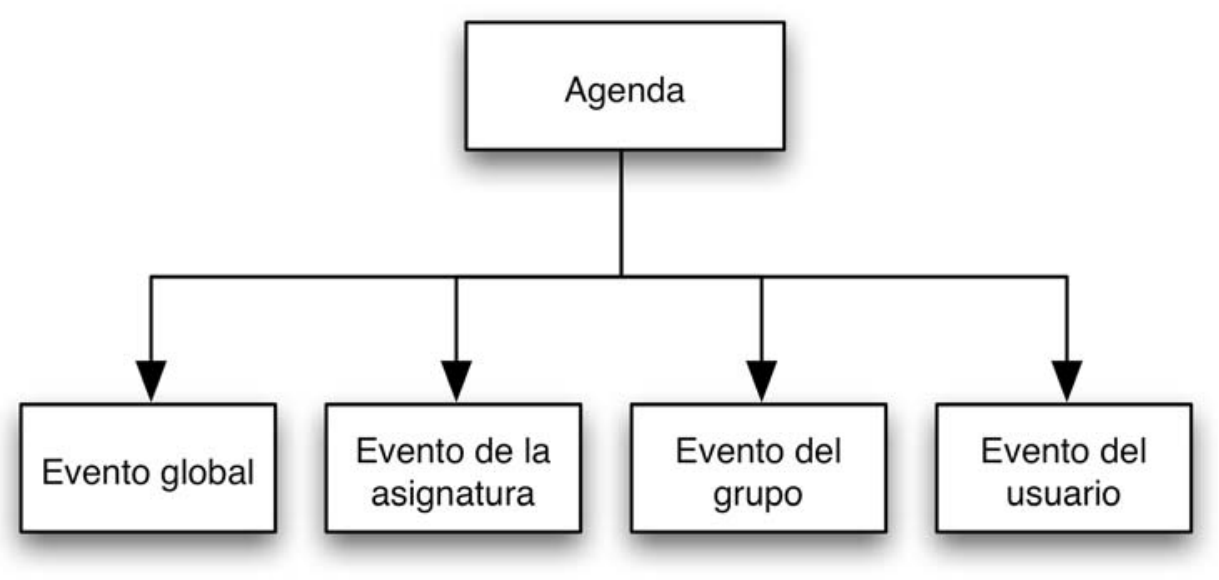

Fig. 4 Agenda para campus virtual de MADEMS

Con estas características la puesta en marcha de la MADEMS a distancia cubre también con el objetivo de formar docentes ad hoc para la sociedad del conocimiento, ya que así aprenderán el uso de la telemática a favor del aprendizaje de sus estudiantes. Debemos recordar aquí que los actuales jóvenes ya crecieron en la sociedad de la información y los medios de comunicación, y están mucho más familiarizados con estas tecnologías que la mayoría de los adultos, incluyendo a sus profesores.

La formación que habrán de recibir les permitirá el empleo de las nuevos medios y recursos para el aprendizaje dentro de sus clases para el desarrollo de proyectos de investigación o para analizar cuestiones de actualidad, como una manera de responder al reto de integrar estas nuevas tecnologías a las lecciones de un modo provechoso y sensato desde el punto de vista educativo. 
Francisco Sierra, Jorge Barojas, Ofelia Contreras y Rina Martínez

DESARROLLO A DISTANCIA DE LA MAESTRÍA EN DOCENCIA PARA LA EDUCACIÓN MEDIA SUPERIOR (MADEMS) DE LA UnivERSidAd NACIONAL AUTÓNOMA DE MÉXico (UNAM)

\section{CONCLUSIONES}

En resumen, las principales acciones estratégicas requeridas para impartir la MADEMS a distancia son las siguientes:

a) Estructurar los contenidos curriculares del plan escolarizado y desarrollar los materiales didácticos de las asignaturas correspondientes a los módulos 1 al 5, implicando la elaboración de guías didácticas para los alumnos y guías para los instructores.

b) Formar los equipos de tutores que apoyen el desarrollo de la MADEMS a distancia.

c) Diseñar y elaborar la plataforma tecnológica para la MADEMS a distancia; está en proceso la propuesta de que tal sea la PLATAFORMA UNAM, en elaboración por la Coordinación de la Universidad Abierta y Educación a Distancia de la UNAM (CUAED) y la Dirección General de Cómputo Académico (DGSCA).

d) Poner en línea todas las asignaturas de la MADEMS para los ocho campos de conocimiento, empezando por el de Biología, el cual opera a nivel piloto en Iztacala desde febrero de 2006, con el apoyo presupuestal de la Secretaría de Desarrollo Institucional de la UNAM.

e) Organizar la estructura operativa a nivel regional para que el módulo 5, pueda impartirse en diferentes instituciones educativas del país. Dado que las prácticas docentes tienen un carácter presencial, para el registro y evaluación de las actividades docentes de los alumnos de la MADEMS se contará con un portafolio electrónico en la plataforma tecnológica instalada para tal efecto.

f) Definir y probar el proceso de ingreso, selección, aceptación e inscripción de alumnos de la MADEMS para la modalidad a distancia, así como organizar el seguimiento, la asesoría y la evaluación de todo el proceso, por parte de la Coordinación de la MADEMS.

Respecto a las posibilidades de generalización y colaboración que ofrece esta Maestría, conviene mencionar que:

(1) Están en proceso las incorporaciones como entidades académicas participantes de la MADEMS, del Centro de Enseñanza para Extranjeros (CEPE) y del Centro de Estudios sobre la Universidad (CESU). También está en proceso la inclusión de los siguientes campos de conocimiento: francés, inglés, letras clásicas y psicología.

(2) La extensión a otros campos de conocimiento en la modalidad a distancia, además del de Biología, permitirá ampliar la cobertura de este posgrado y 
Francisco Sierra, Jorge Barojas, OfElia Contreras y Rina MartíneZ

DESARROLLO A DISTANCIA DE LA MAESTRÍA EN DOCENCIA PARA LA EDUCACIÓN MEDIA SUPERIOR (MADEMS) DE LA UnivERSIDAD NACIONAL AUTÓNOMA DE MÉXICO (UNAM)

apoyarse en establecimiento de convenios con diversas instituciones educativas nacionales, especialmente en relación con la realización de las prácticas docentes, y desarrollar tesis en codirección entre académicos de la UNAM y de otras instituciones. Esta posibilidad es particularmente apropiada para hacer realidad los espacios educativos en los cuales participa la institución.

(3) La creación, operación y ampliación de la MADEMS es un hecho significativo en la UNAM porque ha hecho posible el desarrollo y puesta en marcha de un modelo de programa multidisciplinario en donde convergen los conocimientos de distintos campos del conocimiento y las experiencias prácticas que implican el desarrollo y aplicación de capacidades en el complejo y diverso universo educativo. Es también una experiencia con efecto multiplicador que plantea la necesidad y posibilidad de cambiar estructuras operativas y proyectar a la Universidad, de manera realista y eficiente, hacia formas de organización que respondan a necesidades tales como: la creación de comunidades de aprendizaje con apoyos de tecnología, la gestión del conocimiento para el manejo del capital intelectual de la institución y, como culminación de lo anterior, el funcionamiento del campus virtual de la UNAM.

(4) Finalmente, la MADEMS es un programa de posgrado que contribuye de la manera siguiente a cada una de las cinco ideas clave que conforman la base conceptual de Virtual Educa:

- La educación: poneénfasis en esta función universitaria, apoyándose en la estructura académica de escuelas, facultades, centros e institutos, con el fin de consolidar el Sistema Universitario de Posgrado cuyos atributos son: articulación, multi e interdisciplina, flexibilidad, sistema tutoral y cuerpos colegiados.

- La capacitación: enfoca los estudios de maestría a la formación de profesionales de la docencia en un nivel en donde en México no existen en la actualidad instancias educativas que de manera organizada y sistemática atiendan esta necesidad nacional.

- La innovación: posibilita escenarios de aprendizaje para que, mediante la utilización del potencial de las tecnologías de la información y la comunicación, propicien la consolidación de la educación a distancia en la Universidad y refuercen las modalidades de educación de tipo presencial.

- La cooperación: establece criterios y mecanismos que permitan aprovechar experiencias e iniciativas, así como optimizar el uso de recursos materiales y humanos y de esta manera, apoyados en la evaluación y la vinculación, hagan promisoria una triple integración 
Francisco Sierra, Jorge Barojas, Ofelia Contreras y Rina MartíneZ

DESARROLLO A DISTANCIA DE LA MAESTRÍA EN DOCENCIA PARA LA EDUCACIÓN MEDIA SUPERIOR (MADEMS) DE LA UNIVERSIDAD NACIONAL AUTÓNOMA DE MÉXICO (UNAM)

académica: entre los ciclos del bachillerato, las licenciaturas y el posgrado; entre los campos de conocimiento de las ciencias, las artes y las humanidades; y entre las ciencias cognitivas, la administración y la tecnología.

- El desarrollo: constituye un factor de transformación social al contribuir a que el sector de la población que atiende la EMS obtenga una formación integral como seres humanos mejor preparados para continuar posteriormente con sus estudios profesionales y hasta de posgrado, y en su caso, con aptitudes para desempeñarse adecuadamente en distintas actividades de producción o de servicios.

\section{REFERENCIAS BIBLIOGRÁFICAS}

Aguirre Ocaña, A. M. (1996) Instrumentos para la evaluación de materiales en Proyecto Pated I Vademécum, Aplicaciones Tecnológicas a la enseñanza a Distancia, $2^{\mathrm{a}}$. edición. Pp. 275-303. Madrid-España: Asociación Nacional de Centros de Enseñanza a Distancia (ANCED).

Barberá, E. (2000) Proceso de Estudios en Enseñanza Universitaria a Distancia con Uso de Nuevas Tecnologías. Revista Iberoamericana de Educación a Distancia. 3(1). 9-26.

Barberá, E. (Coord.); Badia, A. y Mominó, J. (2001) La incógnita de la educación a distancia. Barcelona: ICE-Horsori.

Bartolomé, A. (1999) El diseño y la producción de medios para la enseñanza. En Cabero, J., Bartolomé A., Cebrián M., Duarte A., Martínez F., Salinas, J. Tecnología Educativa. Pp. 71-85. Madrid: Síntesis.

Bates, A. (Tony) y Poole, G. (2003). Effective Teaching with Technology in Higher Education. USA: Jossey-Bass.

Bautista Liébana, J. R., Martínez Romero, R., Sainz Ibáñez, M. (2001) La evaluación de materiales didácticos para la educación a distancia. Revista Iberoamericana de Educación a Distancia. 4 (1). 73-95.

Feldman, S., McElroy, E. J, LaCour, N. (2000) Distance Education, Guidelines for Good Practice. New Jersey: American Federation Of Teachers.

García Aretio, L. (1998) "Indicadores para la Evaluación de la Enseñanza en una Universidad a Distancia”. Revista Iberoamericana de Educación a Distancia. 1 (1). 63-85.

García Aretio, L. (1999) Fundamento y Componentes de la Educación a Distancia. Revista Iberoamericana de Educación a Distancia. 2 (2). 43-61.

González Boticario, J., Gaudioso Vázquez E. y Hernández del Olmo, F. (2000) Una Organización de los Recursos de Internet para la Enseñanza a Distancia. Revista Iberoamericana de Educación a Distancia. 3 (1). 51-73.

Harasim, L., Hiltz, S., Teles, L. y Turoff, M. (1995). Learning Networks. A guide to teaching and learning on line. USA: MIT Press. 
Francisco Sierra, Jorge Barojas, Ofelia Contreras y Rina Martínez

DESARROLLO A DISTANCIA DE LA MAESTRÍA EN DOCENCIA PARA LA EDUCACIÓN MEDIA SUPERIOR (MADEMS) DE LA UnivERSIDAD NACIONAL AUTÓNOMA DE MÉXICO (UNAM)

Lévy, P. (1995). ¿Qué es lo virtual? España: Paidós.

Moore, M. y Kearsley, G. (1996). Distance Education. A systems view. USA: Wadsworth Publishing Company.

Pozo J. I. y Monereo C. (1999) El aprendizaje estratégico. Madrid: Aula XXI Santillana.
Spector, J. M. (2001) A Philosophy of Instructional Design for the $21^{\text {st }}$ Century. Journal of Structural Learning and Intelligent Systems. 14. 307-318.

Uden, L. (2003) An Engineering Approach for Online Learning. Journal of Distance Education Technologies. 1 (1) 63-77.

\section{PALABRAS CLAVE}

Postgrado, Formación docente, Educación a distancia.

\section{KEY WORDS}

Graduate program, Teacher's education, Distance education.

\section{PERFIL ACADÉMICO DE LOS AUTORES}

Javier Sierra Vázquez: Físico por la Universidad Nacional Autónoma de México. Maestro en Educación con especialidad en cognición en los procesos de enseñanza-aprendizaje por el Instituto Tecnológico y de Estudios Superiores de Monterrey. Personal Académico del Centro de Instrumentos de la UNAM. Profesor de Física del Sistema Incorporado de la UNAM. Director de Sistemas de Diseño Lógico, S.A. de C.V. Actualmente: Secretario Académico de la Maestría en Docencia para la Educación Media Superior de la UNAM. Correo electrónico: javier.sierra@alexandria21.net

Jorge Barojas Weber: Doctor en Física por la Universidad de París, Francia, Profesor en el Departamento de Física de la UAM - Iztapalapa, Senior Education Fellow de la American Physical Society, Secretario de la International Commission on Physics Education, Director de Educación de la Comisión Nacional para el Ahorro de Energía. Actualmente Profesor en la Facultad de Ciencias y Coordinador de la Maestría en Docencia para la Educación Media Superior, ambas de la UNAM. Correo electrónico: jbarojas@posgrado.unam.mx 
Francisco Sierra, Jorge Barojas, Ofelia Contreras y Rina MartíneZ

DESARROLLO A DISTANCIA DE LA MAESTRÍA EN DOCENCIA PARA LA EDUCACIÓN MEDIA SUPERIOR (MADEMS) DE LA UniversidAd NACIONAL AuTÓNOMA DE MÉXICO (UNAM)

Ofelia Contreras Gutiérrez: Licenciada en Psicología, Maestra en Enseñanza Superior, Doctora en Pedagogía por la UNAM. Profesora Titular B en Desarrollo y Educación, Miembro del Sistema Nacional de Investigadores I. Autora de 3 libros, 15 capítulos de libro, 18 artículos en revistas especializadas, 140 ponencias en eventos académicos especializados nacionales e internacionales. Asesora de 63 tesis de licenciatura y posgrado. Jefa del proyecto de Investigación Evaluación y Planeación Educativa y Responsable de la MADEMS en la FES Iztacala UNAM. Correo electrónico: ofeliaco@ servidor.unam.mx

Rina Martínez Romero: Doctora en Psicología. Facultad de Psicología, Universidad Autónoma de Madrid. Como Profesora de la Facultad de Psicología-UNAM ha impartido asignaturas como, teorías psicológicas de la instrucción, psicología educativa, psicología pedagógica y tecnología de la educación. Actualmente es Asesora en la Secretaría de Desarrollo Institucional-UNAM, en temas de educación y diseño curricular. Es especialista en educación a distancia y en diseño instruccional para la elaboración de materiales didácticos. Correo electrónico: rinam@servidor.unam.mx

Dirección postal:

Universidad Nacional Autónoma de México. $8^{\circ}$ piso Torre de Rectoría.

Ciudad Universitaria. C. P. 04510. México, D. F. México.

Fecha de entrega: 11.07.06

Fecha de aceptación: 20.10.06 